\title{
THE REUTER-LEDERMANN REPRESENTATION FOR BIRTH AND DEATH PROCESSES
}

\author{
EDWARD C. WAYMIRE
}

\begin{abstract}
The identification of the mass of the integrator at zero is made for the integral representation obtained by Reuter and Ledermann for the transition probabilities of birth and death processes. An ergodic theorem is given as an application of this result.
\end{abstract}

1. Introduction. In a fundamental paper on the backward and forward differential equations of the simple birth and death process, W. Ledermann and G. E. H. Reuter (1954) obtained by elementary methods an integral representation for the transition probabilities of the minimal process. Later, S. Karlin and J. L. McGregor (1957a) developed a more complete representation theory by deriving integral representations which are in correspondence with the solutions to certain moment problems. Part of the beauty of these representations lies in the ease with which one can get at the asymptotic behavior of the transition probabilities. In particular, an explicit formula for the limiting distribution can be written down once the mass of the integrator at the origin is known. In the Karlin-McGregor representation the mass of the integrator is obtained by an appeal to the theory of moment problems. However, Ledermann and Reuter left this problem unsolved for their representation [2, p. 345]. In this annoucement we shall identify the mass of the integrator at the origin without going outside the boundaries of the ReuterLedermann exposition; the essential ingredients of which are calculus, linear algebra, and the weak convergence of distributions. This result extends the applicability of the Reuter-Ledermann representation while preserving its remarkable simplicity and accessibility.

2. Notation. The birth and death parameters are $\lambda_{0}, \lambda_{1}, \ldots, \mu_{1}, \mu_{2}, \ldots$ In the case $\lambda_{0}=0$ the problem was solved by Ledermann and Reuter. Consequently, we will assume that all of the parameters are strictly positive real numbers. We shall use exactly the same notation as used in [2], but for ease of reference we shall introduce some of it as we go along.

3. The identification. In the notation of Ledermann and Reuter [2, p. 344], $\rho_{0}$ is the mass of the integrator at zero.

TheOREM. (a) $\rho_{0}=0$ if $\sum_{n=0}^{\infty} \omega_{n} / \lambda_{n}=\infty$.

(b) $\rho_{0}=\left(\lambda_{0}^{2} \sum_{n=0}^{\infty} \omega_{n} / \lambda_{n}\right)^{-1}$ if $\sum_{n=0}^{\infty} \omega_{n} / \lambda_{n}<\infty$, where $\omega_{0}=1, \omega_{n}$ $=\left(\lambda_{1} \cdots \lambda_{n}\right) /\left(\mu_{1} \cdots \mu_{n}\right)$.

Received by the editors October 15, 1975.

AMS (MOS) subject classifications (1970). Primary 60J80, 60-01.

( ) American Mathematical Society 1976 
Proof. The proof of (a) is contained in the work of Ledermann and Reuter [2, p. 345].

It follows from the representation that

$$
\lim _{t \rightarrow \infty} f_{i j}(t)=l_{i-1} m_{j} \phi_{i-1}(0) \rho_{0}=\lambda_{0}^{2} \frac{\omega_{j}}{\lambda_{j}} \cdot \rho_{0},
$$

where $\quad l_{-1}=\lambda_{0}, l_{0}=1, l_{k}=\left(\lambda_{1} \cdots \lambda_{k}\right)^{-1}(k \geqslant 1), \quad$ and $\quad m_{0}=1, m_{k}$ $=\left(\mu_{1} \cdots \mu_{k}\right)^{-1}(k \geqslant 1)$. Since the transition probabilities $\left\{f_{i j}(t)\right\}$ are substochastic, it follows that

$$
\rho_{0} \leqslant\left(\lambda_{0}^{2} \sum_{j=0}^{\infty} \frac{\omega_{j}}{\lambda_{j}}\right)^{-1}
$$

Our job is to show the reverse inequality. Since the integrator, $\rho$, is the weak limit of a sequence, $\left\{\rho^{(n)}\right\}$, whose masses at zero are known [2, pp. 337-340], we can apply Alexandrov's Theorem from the theory of weak convergence of distributions [1, Theorem 2.1, p. 11] to get

$$
\begin{aligned}
\rho_{0} & =\rho(\{0\}) \geqslant \limsup _{n \rightarrow \infty} \rho^{(n)}(\{0\}) \\
& =\limsup _{n \rightarrow \infty}\left(\sum_{k=0}^{n} l_{k-1} m_{k}\left\{\phi_{k-1}\left(\alpha_{0}^{(n)}\right)\right\}^{2}\right)^{-1} .
\end{aligned}
$$

Now, $\phi_{k-1}(x)$ is a polynomial of degree $k$ having $k$ distinct real zeroes with $\alpha_{0}^{(k)}<\alpha_{0}^{(n)}<0$ as its largest zero [2, p. 330]. Moreover, $\phi_{k-1}(0)=\lambda_{0} / l_{k-1}$ $>0$. The $k-1$ zeroes of the derivative of $\phi_{k-1}(x)$ all being accounted for by the Mean Value Theorem of elementary calculus, it now follows that

$$
\begin{array}{r}
0<\phi_{k-1}\left(\alpha_{0}^{(n)}\right)<\phi_{k-1}(0)=\lambda_{0} / l_{k-1} \\
\text { for } k=0,1, \ldots, n .
\end{array}
$$

Substituting this into (1), we get

$$
\rho_{0} \geqslant\left(\lambda_{0}^{2} \sum_{n=0}^{\infty} \frac{\omega_{n}}{\lambda_{n}}\right)^{-1}
$$

As a corollary we can write down the usual ergodic theorem.

Corollary.

$$
\lim _{t \rightarrow \infty} f_{i j}(t)=\omega_{j} / \lambda_{j} \sum_{n=0}^{\infty} \frac{\omega_{n}}{\lambda_{n}}
$$

where the right-hand side is zero if $\sum_{n=0}^{\infty} \omega_{n} / \lambda_{n}=\infty$. 


\section{REFERENCES}

1. P. Billingsley (1968), Convergence of probability measures, Wiley, New York. MR 38 \# 1718.

2. W. Ledermann and G. E. H. Reuter (1954), Spectral theory for the differential equations of simple birth and death processes, Philos. Trans. Roy. Soc. London Ser. A 246, 321-369. MR 15, 625.

3. S. Karlin, and J. L. McGregor (1957a), The differential equation of birth-and-death processes, and the Stieltjes moment problem, Trans. Amer. Math. Soc. 85, 489-546. MR 19, 989.

4. (1956b), The classification of birth and death processes, Trans. Amer. Math. Soc. 86, 366-400. MR 20 \#1363.

Department of Mathematics, University of Arizona, Tucson, Arizona 85721 